\title{
Internaçōes por Pé Diabético: Comparação entre o Custo Direto Estimado e o Desembolso do SUS
}

\begin{abstract}
RESUMO
Foram seguidas 109 internações de pacientes com diabetes e ulcerações nos pés na rede do Sistema Único de Saúde (SUS) em Sergipe, com o objetivo de estimar o custo direto hospitalar e comparar com os valores do desembolso do SUS. Os dados foram coletados desde a admissão até a alta ou o óbito dos casos incluídos, sendo anotadas as características clínicas dos pacientes e os desfechos das internações. Foram calculados o custo direto estimado e o desembolso do SUS. Todos eram portadores de diabetes tipo 2 e a maioria das ulcerações $(64,2 \%)$ foram classificadas como Wagner 4 ou 5 . Evoluíram com alta sem amputação 43 pacientes $(39,4 \%)$ e $52(47,7 \%)$ com alta após amputação. Evoluíram para óbito 14 pacientes (12,8\%). O custo estimado variou de $R \$ 943,72$ a $R \$ 16.378,85$, com média de $R \$ 4.461,04$. O valor do desembolso do SUS variou de $R \$ 96,95$ a $R \$ 2.410,18$, com média de $R \$$ 633,97 , cerca de sete vezes inferior. As menores defasagens entre os custos ocorreram nos pacientes assistidos no Hospital Beneficente e as maiores naqueles tratados com amputações menores. (Arq Bras Endocrinol Metab 2008; 52/3:523-530)
\end{abstract}

Descritores: Custo da doença; Pé diabético; Amputação; SIH SUS; UIceração

\begin{abstract}
In Hospital Care for Diabetic Foot: a Comparison Between the Estimated Cost and the SUS Reimbursement.

This study aimed to analyze costs for treating patients with diabetic foot cared by the public Brazilian Health System (SUS), comparing the estimated cost with the amount of SUS reimbursement. A cohort prospective study carried out in hospitals that provide services for the Unified Health System in Sergipe, involving 109 hospitalization episodes of patients with diabetes and foot ulcers. We follow these patients day by day and estimated the hospital direct cost and the SUS reimbursement. All patients had type 2 diabetes and the majority of ulcers $(64,2 \%)$ were classified as Wagner 4 or 5 . Forty-three $(39,4 \%)$ healed without amputation and fifty-two $(47,7 \%)$ healed with amputation. Fourteen $(12,8 \%)$ patients died. Hospital direct cost ranged from $R \$ 943.72$ to $R \$ 16,378.85$; with an average of $R \$ 4,461.04$. The SUS reimbursement varied from $R \$ 96.95$ to $R \$ 2,410.18$, with an average of $R \$$ 633.97, usually seven times low. Smaller difference between costs occurred in patients from the Beneficent hospital and higher rates occurred in those treated with minor amputation. (Arq Bras Endocrinol Metab 2008; 52/3:523-530)
\end{abstract}

Keywords: Cost of illness; Diabetic foot; Amputation; Ulceration; SIH-SUS

\section{artigo original}

\author{
KaRLA F. REZENDE \\ MARCO A. P. NUNES \\ NaIRA H. Melo \\ Domingos Malerbi \\ ANTÔNIO R. CHACRA \\ MARCO B. FERRAZ
}

Programa de Pós-Graduação em Endocrinologia da

Universidade Federal de São

Paulo (Unifesp) e Centro Paulista de Economia da Saúde (CPES),

São Paulo, SP, Brasil. 


\section{INTRODUÇÃO}

s ulcerações nos pés atingem cerca de $15 \%$ dos pacientes com diabetes melito ao longo da vida e o tratamento dessas feridas é complexo, principalmente daquelas infectadas e com acentuada profundidade, que contribuem para maior possibilidade de amputação (1). Como demonstrou Pecoraro e cols. (2), em 1990, o risco de amputação de membros inferiores em pacientes com diabetes é aproximadamente 40 vezes maior que na população geral. Um estudo realizado no Brasil (3) mostrou que 66,3\% das amputações realizadas em hospitais gerais ocorrem em portadores de diabetes.

$\mathrm{O}$ número de indivíduos com diabetes melito do tipo 2 está crescendo, e, conseqüentemente, também a freqüência das complicações a longo prazo associadas à doença. Esse aumento está relacionado a danos que comprometem produtividade, qualidade de vida e sobrevida dos pacientes, além de envolver um alto custo (4). Dessa forma, uma das grandes preocupações dos sistemas de saúde é o elevado investimento do tratamento e a reabilitação dos doentes com ulcerações dos pés, estimado por Ramsey e cols. (5) em aproximadamente US\$ 28.000 por ano.

No Brasil, a partir de 1990, Pedrosa e cols. (6) iniciaram um trabalho que visava a divulgação do conceito pé diabético com a realização de workshops, o projeto Salvando o pé diabético, com base na bem-sucedida experiência de criação de equipes multidisciplinares nos Estados Unidos e na Europa, resultando capacitação de profissionais da área médica e na conseqüente sensibilização de gestores de saúde por todo o país. A despeito dessa nobre iniciativa, a macroorganização do cuidado individual referente ao pé diabético no país, aparentemente, tem recebido pouca atenção do Ministério da Saúde que inicialmente apoiou o projeto, enviando profissionais aos workshops, mas não assumiu a gestão das ações e recursos necessários ao pleno êxito do projeto, o que acarretaria no controle mais efetivo desse desfecho no Brasil. Publicações nacionais, embora escassas, mostram a precária condição de assistência aos portadores de diabetes no SUS, pois muitos deles não têm informações adequadas ao autocuidado, e poucos têm seus pés examinados nas consultas $(7,8)$, havendo elevadas taxas de internações, amputações e mortes por este agravo em vários lugares do país (9-13).

Pelos motivos expostos, realizamos uma avaliação prospectiva de internações de pacientes com diabetes e úlceras nos pés na rede SUS de Sergipe, com o objetivo de estimar os custos diretos relacionados às hospitalizações e compará-los com o desembolso do SUS, assim como analisá-los de acordo com o desfecho clínico da internação.

\section{MATERIAL E MÉTODOS}

A pesquisa foi realizada de acordo com a Resolução no 196/1996 do Conselho Nacional de Saúde e com a Declaração de Helsinque e teve aprovação do Comitê de Ética em Pesquisa da Universidade Federal de Sergipe (UFS).

Foram incluídos neste trabalho e acompanhados durante as internações pacientes com diabetes e úlceras nos pés assistidos nos serviços hospitalares do SUS, no Estado de Sergipe, que prestam atendimento aos portadores de pé diabético, sendo um do tipo fundação beneficente, um hospital público estadual e um hospital público federal. No período da pesquisa, não havia na cidade outra porta de entrada nessas instituições que não fossem os pronto-socorros e, a partir deles, os pacientes foram internados nas enfermarias cirúrgicas ou clínicas, recebendo cuidados por equipes de clínicos ou cirurgiões gerais não especializados na abordagem do pé diabético. Dessa forma, a maneira utilizada para a convocação dos pacientes para a pesquisa foi uma busca diária e aleatória destes por meio de consulta às equipes de enfermagem. Assim, ficavase ciente das novas admissões que satisfizessem os critérios de inclusão do estudo.

O grupo de pesquisa foi formado por 40 acadêmicos de medicina, subdivididos em cinco equipes, encarregado de acompanhar a evolução diária dos pacientes, desde o momento da internação até o da alta hospitalar ou do óbito. Após esclarecimento sobre os objetivos do estudo e a obtenção de seu consentimento, era realizado exame clínico do paciente e observados prescrições, exames complementares e procedimentos a que os pacientes tivessem sido submetidos nesse período, informações que eram registradas em formulário padronizado. Algumas das variáveis analisadas foram: tempo de duração e tipo do diabetes melito, presença ou não de comorbidades associadas, tipo de tratamento da doença, número de internações anteriores e nível de amputação. Além disso, as úlceras foram graduadas quanto à gravidade no momento da hospitalização, conforme a classificação de Wagner (Tabela l) (14). 
Tabela 1. Classificação de Wagner para as lesões do pé diabético.

\begin{tabular}{ll}
\hline Grau & Características \\
Grau 0 & Pé em risco \\
\hline Grau 1 & Úlcera superficial, sem infecção \\
\hline Grau 2 & Profunda, com infecção sem osteomielite \\
\hline Grau 3 & Profunda, abscesso com osteomielite \\
\hline Grau 4 & $\begin{array}{l}\text { Necrose localizada em dedos, região } \\
\text { plantar ou calcanhar }\end{array}$ \\
\hline Grau 5 & Necrose de quase todo o pé \\
\hline
\end{tabular}

Foram excluídos da pesquisa os pacientes nos quais o diagnóstico da ulceração ocorreu durante a internação, não sendo esta a causa da procura do cuidado hospitalar, aqueles que foram transferidos para outro hospital ou não tiveram o diagnóstico de diabetes confirmado, segundo os critérios da Organização Mundial da Saúde (OMS) (15), assim como aqueles que tinham seus dados incompletos.

A cicatrização primária foi caracterizada pela melhora ou resolução da ferida sem a necessidade de amputação, definida como ressecção transversal da parte terminal do membro e classificadas em menor e maior, utilizando a articulação do tornozelo como referência, sendo distal e proximal a esta, respectivamente (16).

$\mathrm{Na}$ análise dos custos, foram considerados os recursos consumidos pelos pacientes durante as internações. Foram estes divididos em diárias hospitalares, medicações, serviços profissionais e exames complementares; denominou-se este de custo estimado. Para esse fim, foram atribuídos valores em reais ( $\mathrm{R} \$$ ) a todos os recursos utilizados no tratamento de tais feridas durante as internações. O valor da diária hospitalar (água, eletricidade, limpeza, lavanderia, recursos humanos, segurança, nutrição e taxas hospitalares) foi estimado por meio das planilhas de custos referentes ao ano de 2003 de cada hospital envolvido. Levou-se em consideração o montante monetário destinado ao suporte das enfermarias, considerando o número de leitos disponíveis e o percentual de ocupação de $80 \%$ ao longo do ano. Os custos dos medicamentos foram estabelecidos a partir da tabela Brasíndice do ano de 2003, calculando-se a média dos três menores valores referentes a cada medicação, para cada hospital. O cálculo do valor dos serviços profissionais, clínicos e cirúrgicos, e dos exames complementares foi feito pela tabela da Associação Médica Brasileira (AMB) de 1996, uma vez que o corpo médico clínico não possuía relação de vínculo empregatício com as instituições envolvidas.

Já os valores desembolsados pelo SUS para o tratamento desses pacientes, o custo SUS, foi calculado por meio da soma dos valores referentes a serviços hospitalares $(\mathrm{SH})$, serviços profissionais $(\mathrm{SP})$, serviços avançados de diagnóstico e terapêutica (SADT), serviços em unidade de terapia intensiva (UTI), serviços referentes a analgesia e ao uso de sangue e derivados, de acordo com o Manual do Sistema de Informações Hospitalares do SUS de 2003 (17).

Considerou-se ainda a variável "permanência a maior", definida como a hospitalização em que o período de internação do paciente ultrapassasse o dobro da permanência prevista na Tabela de Procedimentos do SUS (17). Os custos indiretos de tais tratamentos, definidos como aqueles decorrentes da perda de produtividade e aposentadoria, não foram calculados, pois não fizeram parte dos objetivos deste estudo.

O registro no banco de dados e a análise estatística foram realizados com a utilização dos programas Microsoft Excel e SPSS 11. Na comparação das variáveis, utilizamos a ANOVA, o teste de Tukey e o teste quiquadrado para as variáveis com distribuição normal e o teste não paramétrico de Mann-Whitney para aquelas sem distribuição normal. Considerou-se estatisticamente significante o valor de $\mathrm{p}<0,05$.

\section{RESULTADOS}

\section{Dados sociodemográficos}

Entre julho e dezembro de 2003, foram acompanhadas 122 hospitalizações. Em 109 casos $(89,3 \%)$, os dados estavam completos e de acordo com os critérios de inclusão, perfazendo o total de 100 pacientes que realizaram 109 internações durante este período - nove pacientes $(8,2 \%)$ internaram duas vezes. Todos eram portadores de diabetes tipo 2 com ulcerações em membros inferiores, que procuraram assistência em hospitais do SUS, em Sergipe. A média de idade do grupo foi de $62,7 \pm 12,2$ anos, variando de 37 a 98 anos. Quando os pacientes foram distribuídos por faixa etária, houve 
maior concentração de indivíduos (78\%) entre a sexta e a oitava décadas de vida $(\mathrm{p}<0,01)$. Quanto à distribuição por sexo, $64(58,7 \%)$ pacientes eram do sexo feminino e a maioria dos indivíduos, ou seja, 73 (67\%) residiam em municípios do interior de Sergipe, ainda que $24(22 \%)$ morassem na capital, Aracaju, e 12 (11\%) procedessem de estados vizinhos. O tempo de ulceração antes da procura da assistência hospitalar variou de 3 a 730 dias, com média de $81,98 \pm 120,28$ dias, sendo $10(9,2 \%)$ pacientes classificados como portadores de ulcerações grau 1 de Wagner; 24 (22\%) como grau 2; cinco $(4,6 \%)$ como grau $3 ; 50(45,9 \%)$ como grau 4 ; e $20(18,3 \%)$ como grau 5 .

As internações ocorreram 70 vezes $(64,2 \%)$ na Fundação Hospitalar Beneficente, 24 vezes $(22 \%)$ no Hospital Público Estadual e 15 vezes $(13,8 \%)$ no Hospital Universitário Federal (Tabela 2). O tempo médio de internação foi de $16,8 \pm 12$ dias, com variação de três a 65 dias, localizando-se nas enfermarias de clínica médica, 40 internações (36,7\%), e de clínica cirúrgica, 69 (63,3\%) internações. O tempo de internação variou com os desfechos dos pacientes, sendo significantemente mais prolongado no grupo submetido à amputação menor $(22,2$ $\pm 19,1$ dias) do que nos pacientes tratados com amputação maior $(12,1 \pm 5,5$ dias $)(\mathrm{p}<0,01)$. A utilização da UTI foi necessária em nove $(8,3 \%)$ casos. A ocorrência da permanência a maior, sugerida pela tabela do SUS, fez-se presente em $76(69,7 \%)$ internações, sendo significativamente mais freqüente quando os pacientes se internavam nas enfermarias de clínica médica $(\mathrm{p}=0,02) \mathrm{e}$ no Hospital Universitário Federal $(\mathrm{p}<0,001)$. Em nenhum dos três hospitais havia disponibilidade de serviços de reabilitação para os pacientes (Tabela 2).

\section{Dados sobre os desfechos das internações e da análise dos custos}

Em 95 (87,2\%) internações seguidas, os pacientes receberam alta hospitalar e, em 14 (12,8\%), os pacientes evoluíram para óbito. Os pacientes foram submetidos a várias abordagens terapêuticas, porquanto 16 (15\%) foram tratados exclusivamente com curativos e tratamento tópico, e $93(85 \%)$ necessitaram de alguma intervenção cirúrgica. Dos pacientes submetidos ao tratamento cirúrgico, $58(62,4 \%)$ sofreram amputações, $35(37,6 \%)$ foram submetidos a debridamentos. Realizaram procedimento de revascularização fêmoro-poplítea cinco $(4,6 \%)$ pacientes. As amputações foram menores em $26(44,8 \%)$ e maiores em $32(55,2 \%)$ dos casos. As úlceras mais profundas, graus 4 e 5 na classifi-
Tabela 2. Caracteristicas clínico-epidemiológicas dos pacientes diabéticos portadores de úlceras nos pés internados em três dos hospitais da rede SUS em Segipe

\begin{tabular}{lr}
$\begin{array}{l}\text { Caracteristicas } \\
\text { Sexo }\end{array}$ \\
Masculino \\
\hline Feminino \\
\hline Procedência & $45(41,3)$ \\
\hline Aracaju & $64(58,7)$ \\
\hline Interior & \\
\hline Outros Estados & $24(22)$ \\
\hline Fluxo prévio & $73(67)$ \\
\hline Espontâneo & $12(11)$ \\
\hline UBS & \\
\hline Centro de especialidade & $31(28,4)$ \\
\hline Tratamento DM & $57(52,3)$ \\
\hline Sem tratamento & $21(19,3)$ \\
\hline Dieta & $15(13,8)$ \\
\hline Droga oral & $8(7,3)$ \\
\hline Insulina & $41(37,6)$ \\
\hline Droga oral + insulina & $42(38,5)$ \\
\hline Classificação de Wagner & $3(2,8)$ \\
\hline Grau 1 & $50(45,9)$ \\
\hline Grau 2 & $20(18,3)$ \\
\hline Grau 3 & $10(9,2)$ \\
\hline Grau 4 & $24(22)$ \\
\hline Grau 5 & \\
\hline Públiblico federal & \\
\hline
\end{tabular}

cação de Wagner, estavam associadas mais freqüentemente ao desfecho de amputação $(\mathrm{p}<0,01)$. Ao se analisar os desfechos dos pacientes, observou-se que 43 $(39,4 \%)$ obtiveram alta após cicatrização primária, 23 $(21,1 \%)$ após amputações menores, $29(26,6 \%)$ após amputações maiores e 14 (12,8\%) evoluíram para óbito. As causas mais freqüentes de óbito foram septicemia e eventos cárdio e cerebrovasculares.

O custo estimado por internamento nesses hospitais foi, em média, de $\mathrm{R} \$ 4.461,04 \pm 2.995,30$, variando de $\mathrm{R} \$ 943,72$ a R\$ 16.378,85, com mediana de R $\$ 3.754,44$. O custo diário foi de R $\$ 265,53$, e o custo total das 109 
internações atingiu a cifra de $\mathrm{R} \$ 486.253,72$. As despesas hospitalares foram responsáveis pela maior parte destes custos $(57 \%)$, seguidas pelas despesas com honorários médicos $(23 \%)$, medicações (17\%) e exames complementares $(3 \%)$. Analisando os valores pagos por internação pelo SUS, o custo SUS, a média por internação foi de R\$ $633,97 \pm 4.945,00$, variando de $\mathrm{R} \$ 96,95$ a $\mathrm{R} \$ 2.410,18$, com mediana de R $\$ 503,41$. O custo diário foi de R\$ 37,74; e o custo total das 109 internações, de R\$ 69.103,03, visto que os serviços hospitalares foram responsáveis por $70 \%$, os serviços profissionais por $27 \%$ e os serviços adicionais de diagnóstico e terapêutica por $3 \%$ deste montante. A diferença entre o custo estimado das internações e o valor do desembolso do SUS foi em média de R $3.021,96 \pm 2.790,02$ por caso, variando de $\mathrm{R} \$$ 771,35 a R\$ 15.426,84 (Tabela 3). Dessa forma, observase que o valor estimado das internações por ulcerações de membros inferiores foi, em média, 7,03 vezes maior que aquele desembolsado pelo SUS. Os percentuais das defasagens entre os valores do custo estimado e do custo SUS atingiram os diferentes componentes das despesas de maneira bastante homogênea: despesas hospitalares de $85,14 \%$, serviços adicionais de diagnóstico e terapêutica de $86,35 \%$ e serviços profissionais de $81,83 \%$.

Ao se analisar os valores dos custos entre as instituições hospitalares envolvidas no estudo, o custo estimado foi significantemente inferior na Fundação Hospitalar Beneficente $(\mathrm{p}<0,01)$ em relação aos Hospitais Públicos Estadual e Federal, já o custo SUS não apresentou diferença significativa entre as instituições. Dessa forma, a diferença de valores entre o custo direto estimado e o que foi desembolsado pelo SUS foi significantemente menor no hospital beneficente, quando comparado aos demais $(\mathrm{p}<0,01)$; mesmo assim, atingiu, no final do estudo, o montante de R\$210.781,72 (Tabela 3).

$\mathrm{Na}$ análise dos custos, de acordo com os desfechos hospitalares, os pacientes que foram tratados com amputação maior apresentaram custo estimado significantemente mais baixo que aqueles submetidos à amputação menor $(\mathrm{p}=0,02)$. Paradoxalmente, o desembolso do SUS foi significantemente mais alto no grupo tratado com amputação maior do que no grupo com cicatrização primária ou submetido a amputação menor $(\mathrm{p}<0,001)$. Houve, assim, tendência de que a maior defasagem entre custo estimado e custo SUS ocorresse nos pacientes tratados com amputações menores $(\mathrm{p}<0,064)$ (Tabela 4$)$.

\section{DISCUSSÃo}

Estudos provenientes de diferentes estados do Brasil têm relatado elevadas taxas de amputação e óbito intra-hospitalar na população com diabetes e úlceras nos pés assistida pelo SUS (9-13). Nosso estudo analisou o cuidado hospitalar, avaliando toda a rede SUS de um estado, e não apenas uma unidade hospitalar como já fora publicado, dando uma noção da qualidade das ofertas disponibilizada pelo SUS a estes indivíduos. Observamos que os pacientes incluídos apresentavam longo tempo de ulceração antes da admissão hospitalar e que cerca de $80 \%$ desses vinham diretamente das unidades básicas de saúde ou

Tabela 3. Custo estimado, custo SUS e diferença entre os custos, em reais (R\$), das 109 internações por pé diabético, de acordo com o tipo de hospital da rede SUS em Sergipe.

\begin{tabular}{|c|c|c|c|c|}
\hline Tipo de hospital & Parâmetro & Custo estimado & Custo SUS & Diferença custo real e SUS \\
\hline $\begin{array}{l}\text { Hospital Beneficente } \\
N=70\end{array}$ & $\begin{array}{l}\text { Média } \\
\pm \text { DP } \\
\text { Soma }\end{array}$ & $\begin{array}{r}3.739,33^{* / * *} \\
2.237,74 \\
261.753,58\end{array}$ & $\begin{array}{r}654,68 \\
519,13 \\
45.827,77\end{array}$ & $\begin{array}{r}3.011,16^{* / * *} \\
1.992,97 \\
210.781,72\end{array}$ \\
\hline $\begin{array}{l}\text { Hospital Público } \\
\text { Federal } \\
\mathrm{N}=15\end{array}$ & $\begin{array}{l}\text { Média } \\
\pm \text { DP } \\
\text { Soma }\end{array}$ & $\begin{array}{r}6.633,67 \\
4.331,25 \\
99.505,06\end{array}$ & $\begin{array}{r}561,44 \\
352,46 \\
8.421,63\end{array}$ & $\begin{array}{r}5.989,32 \\
4.173,24 \\
89.839,85\end{array}$ \\
\hline $\begin{array}{l}\text { Hospital Público } \\
\text { Estadual } \\
\mathrm{N}=24\end{array}$ & $\begin{array}{l}\text { Média } \\
\pm \text { DP } \\
\text { Soma }\end{array}$ & $\begin{array}{r}5.208,12 \\
3.231,40 \\
124.995,08\end{array}$ & $\begin{array}{r}618,90 \\
511,21 \\
14.853,63\end{array}$ & $\begin{array}{r}4.397,34 \\
2.944,28 \\
105.536,19\end{array}$ \\
\hline $\begin{array}{l}\text { Total } \\
N=109\end{array}$ & $\begin{array}{l}\text { Média } \\
\pm \text { DP } \\
\text { Soma }\end{array}$ & $\begin{array}{r}4.461,04 \\
2.995,33 \\
486.253,72\end{array}$ & $\begin{array}{r}633,97 \\
494,97 \\
69.103,03\end{array}$ & $\begin{array}{r}3.726,21 \\
2.790,02 \\
406.157,76\end{array}$ \\
\hline
\end{tabular}


Tabela 4. Tempo de internação, custo estimado, desembolso do SUS e diferença entre os custos, de acordo com o desfecho das 109 hospitalizações por pé diabético em Sergipe.

\begin{tabular}{|c|c|c|c|c|}
\hline & \multicolumn{4}{|c|}{ DESFECHO DA INTERNAÇÂO } \\
\hline & $\begin{array}{l}\text { Cicatrização primária } \\
\qquad N=43\end{array}$ & $\begin{array}{l}\text { Alta com amputação } \\
\text { menor } \\
N=23\end{array}$ & $\begin{array}{c}\text { Alta com amputação } \\
\text { maior } \\
N=29\end{array}$ & $\begin{array}{l}\text { Óbito } \\
N=12\end{array}$ \\
\hline $\begin{array}{l}\text { Custo estimado } \\
\text { (R\$) }\end{array}$ & $4.128,6 \pm 2.205,6$ & $5.679,4^{*} \pm 4.734,2$ & $3.528,8 \pm 1.414,5$ & $5.411,5 \pm 873,3$ \\
\hline Custo SUS (R\$) & $426,1 \pm 489,6$ & $611,5 \pm 423,1$ & $844,3^{\star \star} \pm 167,1$ & $873,5 \pm 770,5$ \\
\hline $\begin{array}{l}\text { Diferença entre } \\
\text { os custos (R\$) }\end{array}$ & $3.493,5 \pm 1.871,3$ & $5.073,8 \pm 4.473,9$ & $2.650,8^{\#} \pm 1.292,2$ & $4.454,8 \pm 3.056,4$ \\
\hline
\end{tabular}

Valores em médias \pm DP. ${ }^{*} \mathrm{p}=0,02 \times$ alta com amputação maior; ** $\mathrm{p}<0,001 \times$ cicatrização primária e alta com amputação menor; ${ }^{*} \mathrm{p}<0,064 \times$ alta com amputação menor.

procuravam espontaneamente as unidades hospitalares, em busca de cuidado adequado, não tendo acessado previamente uma unidade de atenção especializada em feridas. Quando analisados os desfechos dessas 109 internações, mais da metade dos pacientes evoluiu com algum tipo de amputação e, destas, 56,1\% foram maiores, enquanto, em cerca de $13 \%$ dos casos, houve óbito. Embora já tenha sido demonstrado que há diferenças significativas nas incidências de amputações em doentes com diabetes no mundo, essas são taxas realmente elevadas e comparáveis àquelas encontradas em países subdesenvolvidos, onde o planejamento e a organização do cuidado referente ao pé diabético ainda não foram implementados (18-20). Já os indicadores provenientes de países desenvolvidos, em que as estratégias para redução das amputações vêm sendo aplicadas, mostram que os portadores de diabetes que apresentem úlceras nos pés evoluem com alguma amputação em $11 \%$ a $29 \%$ das vezes, sendo a maioria constituída de amputações menores $(21,22)$.

$\mathrm{O}$ custo direto estimado por internação foi de R\$ $4.461,04$, enquanto a média do valor pago pelo SUS para esse mesmo grupo de doentes foi de R\$ 633,97; presumindo-se que há déficit médio em torno de $\mathrm{R} \$$ 3.700,00 por internação. Dessa forma, o valor pago pelo SUS foi, em média, sete vezes menor que o custo estimado das internações desses pacientes para as instituições hospitalares, e a defasagem de valores, quando consideradas as 109 internações, atingiu o total de R\$ 406.157,76. É interessante considerar que essa diferença de valores foi significantemente maior nos pacientes que foram tratados com amputações menores em relação aos que só realizaram curativos ou foram submetidos a amputações maiores, provavelmente em razão do tempo de internação mais prolongado nos primeiros, já que as despesas com as diárias hospitalares foram responsáveis pela maior parte dos gastos totais. Milman e cols. (11), em 2001 , introduziram a análise do custo hospitalar de pacientes com pé diabético no Brasil em um conjunto hospitalar em Sorocaba, SP e, considerando a tabela do Sistema de Informações Hospitalares do Sistema Único de Saúde (SIH-SUS), encontraram valores que variaram de $\mathrm{R} \$ 211,16$ a $\mathrm{R} \$ 7.164,00$ com mediana de R\$ $1.004,59$. Esses valores foram superiores aos encontrados no presente estudo: R\$ 503,4l. Essa diferença pode ser explicada pela oferta tecnológica mais qualificada em Sorocaba, uma vez que $30 \%$ dos pacientes foram submetidos a procedimentos de revascularização, sabidamente onerosos, em relação ao achado de 4,6\% da nossa pesquisa. $\mathrm{O}$ valor mediano dos nossos casos submetidos a procedimento de revascularização foi de R\$ 1.118,20. A inexistência de reabilitação para os pacientes seguidos por nosso grupo e a possibilidade de subsídios com a tabela SIH-SUS para o Conjunto Hospitalar de Sorocaba, uma vez que se trata de um conjunto de hospitaisescola, também devem ser considerados para explicar as diferenças entre os valores encontrados.

Considerando a permanência a maior, cerca de 70\% das internações nos pacientes estudados excederam esse limite, o que concorda com os achados de Milman e cols. (11) que também relataram que a maioria das internações se prolongou além do tempo máximo estabelecido pelo SUS. O tempo médio de internação encontrado 
no nosso estudo é comparável ao do estado da Califórnia, EUA que, por sua vez, apresenta tempo de internação significativamente mais curto que o da Holanda (23), que é de 41 dias, e o da Espanha (24) que chegou a 51 dias. Curiosamente, estes dois últimos centros relataram menor freqüência de amputações em relação à Califórnia e ao presente estudo, sugerindo que, para a preservação do membro, talvez sejam necessários cuidados que exijam períodos de internação mais prolongados para alguns doentes. O que confirma um fato importante constatado nos dados desta pesquisa: alguns pacientes apresentando úlceras supostamente mais graves e, por isso, com indicação de cuidado mais prolongado e complexo, acabaram submetidos precocemente a amputações maiores, permanecendo internados por menor período. Isso é relevante, pois se reflete aparente menor custo financeiro direto para a instituição hospitalar que está cuidando do paciente, em detrimento de maiores custos sociais, humanos e também financeiros para o sistema de saúde, a sociedade e o próprio doente.

Este estudo tem algumas limitações, deve-se considerar que o Brasil é um país de extensão continental e poderá haver relevante diferença na qualidade tecnológica das ofertas disponibilizadas pelo SUS aos portadores de pé diabético em diferentes regiões do país. Outro ponto importante é que o estudo limitou-se apenas à avaliação do custo direto intra-hospitalar, para o cálculo do custo total das ulcerações teriam que ser acrescidas as despesas com o cuidado ambulatorial das feridas, assim como do cuidado domiciliar e os custos indiretos, o que incluiria os prejuízos decorrentes da perda de produtividade, o valor dos recursos gastos com suporte de previdência por aposentadoria ou morte prematura, além do tratamento das repercussões psicológicas que uma amputação acarreta para o paciente e seus familiares. Na valoração dos honorários médicos, assim como dos exames complementares, os quais são reconhecidamente de difícil mensuração, utilizamos tabela sugerida por instituição de representação nacional, no caso a tabela da AMB. Embora esta se referira ao valor a ser cobrado por serviço prestado, seguimos a tendência da literatura internacional $(4,5,18,21,25)$ em utilizar tabelas de abrangência nacional para estimar esses recursos.

Diante da crescente preocupação com a redução das taxas de amputações em pacientes diabéticos em muitos países, desde 1990, a OMS, por meio da Declaração de Saint Vincent (26), sugere estratégias preventivas e terapêuticas, a fim de reduzir as amputações em $50 \%$ no mundo até o século XXI; além disso, instituições inter- nacionais têm publicado sugestões e consensos que visam a estratificação dos pacientes e a organização das ofertas e dos cuidados nos sistemas de saúde (27).

A promulgação da Constituição de 1988 (28) garantiu o direito à saúde para todos os cidadãos brasileiros $\mathrm{e}$ instituiu o SUS. Segundo os artigos 195 e 198 da Constituição Federal, o financiamento do SUS é de responsabilidade comum da União, dos Estados, do Distrito Federal e dos Municípios. A esfera federal ainda é a responsável pela maior parcela do financiamento do SUS, embora se estime que a participação dos municípios venha crescendo ao longo dos últimos anos. O SUS utiliza o SIH/SUS para o desembolso dos serviços prestados pelas unidades hospitalares públicas, beneficentes e privadas contratadas para a prestação de serviços, com base em tabela própria de procedimentos. Todo o sistema público utiliza uma única tabela de preços definida pelo Ministério da Saúde, para pagamento aos prestadores de serviços. Este trabalho mostra a dimensão da defasagem entre os valores desembolsados pelos hospitais e o montante pago pelo SUS para o cuidado dos portadores de pé diabético, provavelmente refletindo o que ocorre no tratamento de outras doenças, deixando óbvia a ambigüidade do Ministério da Saúde, que, oficialmente, é o responsável pela eficiência do serviço prestado e pela sustentabilidade econômica das instituições aptas e interessadas a prestar seus serviços ao SUS, porém, na prática, o Ministério se comporta de modo inadequado, reembolsando parcialmente as despesas hospitalares e patrocinando uma precária qualidade de assistência, nesta pesquisa evidenciada pela elevada taxa de amputações maiores e óbitos.

A economia da saúde é uma área que envolve tanto conceitos e conhecimentos da medicina, como princípios da economia; tem o potencial de contribuir para a visibilidade do impacto das doenças pelos gestores de saúde. Esses resultados sugerem a reflexão e a revisão, por parte do Ministério da Saúde, do gerenciamento e do pagamento das instituições prestadoras de serviços ao SUS; assim como do planejamento do cuidado integral com o portador de diabetes, o que envolveria a inclusão de ações educativas e do exame dos pés dos pacientes na rotina das unidades de atenção básica à saúde e a criação de ambulatórios especializados no tratamento de feridas, para onde seriam referenciados os casos de pé em risco de ulceração ou já ulcerados. Estas medidas foram eficazmente aplicadas em vários países desenvolvidos (29-32), e também pelo grupo de Pedrosa e cols. no Brasil (6), acarretando diminuição significativa das taxas de amputações e dos custos relacionados ao pé diabético. 


\section{AGRADECIMENTOS}

Este estudo foi parcialmente financiado pelo Programa de Apoio a Núcleos de Aperfeiçoamento de Pessoal de Nível Superior (Capes). Agradecimento especial aos funcionários das instituições envolvidas no estudo pela ajuda na aquisição dos dados hospitalares. Agradecimento ao Dr. Marco Aurélio Prado Nunes, Secretaria Municipal da Saúde de Aracaju, pela ajuda nos cálculos envolvendo a tabela SIH-SUS.

\section{REFERÊNCIAS}

1. Reiber GE. The epidemiology of diabetes foot problems. Diabet Med. 1996;13 Suppl 1:S6-11.

2. Pecoraro RE, Reiber GE, Burgess EM. Pathways to diabetic limb amputation. Basis for prevention. Diabetes Care. 1990;13(5):513-21.

3. Gamba MA. Amputações por diabetes mellitus, uma prática prevenível? Acta Paul Ent. 1998;11(3):92-100.

4. King $\mathrm{H}$, Aubert RE, Herman WH. Global burden of diabetes, 1995-2025: prevalence, numerical estimates, and projections. Diabetes Care. 1998;21(9):1414-31.

5. Ramsey SD, Newton K, Blough D, et al. Incidence, outcomes, and cost of foot ulcers in patients with diabetes. Diabetes Care. 1999;22(3):382-7.

6. Pedrosa HC, Leme LAP, Novaes C, Saigg M, Sena F, Gomes EB, et al. The diabetic foot in south America: progress with the brazilian save the diabetic foot project. International. Diabetes Monitor. 2004;16(4):17-23.

7. Ministério da Saúde/Organização Pan Americana de Saúde: avaliação do Plano de Reorganização da Atenção à Hipertensão Arterial e ao Diabetes Mellitus no Brasil. Brasília: Ministério da Saúde (2004)

8. Calsolari MR, Castro RF, Maia FCP, Castro AV, Reis R, Ferreira $A R$, et al. Análise retrospectiva dos pés de pacientes diabéticos do ambulatório de diabetes da Santa Casa de Belo Horizonte, MG. Arq Bras Endocrinol Metab. 2002; 46(2):173-6.

9. Pitta GBB, Castro AA, Soares AMMN, Maciel CJJ, Silva LDM, Muniz VMT, et al. Profile of patients with diabetic foot receiving care at the Hospital Escola José Carneiro and at the Unidade de Emergência Armando Lages. J Vasc Br. 2005;4(1):5-10.

10. Jorge BH, Borges MF, Brito VN, Santos TGM, Thirone AC. Análise clínica e evolução de 70 casos de lesões podais infectadas em pacientes diabéticos. Arq Bras Endocrinol Metab. 1999;43(5):366-72.

11. Milman MHSA, Leme CBM, Borelli DT, Kater FR, Baccili ECDC Rocha RCM, et al. Pé diabético: avaliação da evolução e custo hospitalar de pacientes internados no Conjunto Hospitalar de Sorocaba. Arq Bras Endocrinol Metab. 2001;45(5):447-51.

12. Brasileiro JL, Oliveira WTP, Monteiro LB, Chen J, Pinho Jr. EL, Molkenthin S, et al. Diabetic foot: clinical aspects. J Vasc Br. 2005;4(1):11-21

13. Cunha EF, Marques EP, Gomes MB. Perfil de pacientes diabéticos internados em Hospital Universitário do Rio de Janeiro. Arq Bras Endocrinol Metab. 1995;39(2):111-15.

14. Wagner FW. The disvascular foot: a system for diagnosis and treatment. Foot and Ankle. 1981;2(2):64-122.
15. World Health Organization: definition, diagnosis and classification of diabetes mellitus and its complications. Report of a WHO consultation, Geneva, World Health Org. (1999).

16. The LEA Study Group. Comparing the incidence of lower extremity amputations across the world: the global lower extremity amputations study. Diabetic Med. 1995;12:14-8.

17. Ministério da Saúde. Manual do Sistema de Informações Hospitalares do SUS (SIH/SUS) 2003.

18. Grupo de Trabalho Internacional sobre Pé Diabético. Consenso internacional sobre o pé diabético. Brasília: Secretaria de Estado de Saúde do Distrito Federal (2001).

19. Gulam-Abbas Z, Lutale JK, Morbach S, Archibald LK. Clinical outcome of diabetes patients hospitalized with foot ulcers, Dar es Salaam, Tanzania. Diabet Med. 2002;19(7):575-9.

20. Hennis AJ, Fraser HS, Jonnalagadda R, Fuller J, Chaturvedi N. Explanations for the high risk of diabetes-related amputation in a Caribbean population of black african descent and potential for prevention. Diabetes Care. 2004;27(11):2636-41.

21. Apelqvist J, Ragnarson-Tennvall G, Persson U, Larsson J. Diabetic foot ulcers in a multidisciplinary setting. An economic analysis of primary healing and healing with amputation. $J$ Intern Med. 1994;235(5):463-71.

22. Jeffcoate WJ, Harding KG. Diabetic foot ulcers. Lancet. 2003;361(9368):1545-51.

23. van Houtum WH, Lavery LA. Outcomes associated with diabetes-related amputations in The Netherlands and in the state of Califórnia, USA. J Intern Med. 1996;240(4):227-31.

24. Calle-Pascual $A L$, Redondo MJ, Ballesteros $M$, et al. Nontraumatic lower extremity amputations in diabetic and non-diabetic subjects in Madrid, Spain. Diabetes Metab. 1997;23(6):519-23.

25. Ragnarson TG, Apelqvist J. Health-economic consequences of diabetic foot lesions. Clin Infec Dis. 2004;39(Suppl 2):S132-9.

26. World Health Organization. Diabetes care and research in Europe: the St. Vincent Declaration. Diabet Med. 1990;7:360.

27. Schaper NC, Apelqvist J, Bakker K. The international consensus and practical guidelines on the management and prevention of the diabetic foot. Curr Diab Res. 2003;3(6):475-9.

28. Constituição da República Federativa do Brasil. Brasília: Senado Federal 1988. 292 p.

29. Larsson J, Apelqvist J, Agardh CD, Stenstrom A. Decreasing incidence of major amputation in diabetic patients: a consequence of a multidisciplinary foot care team approach? Diabet Med. 1995;12(9):770-6.

30. Driver VR, Madsen J, Goodman RA. Reducing amputation rates in patients with diabetes at a military medical center: the limb preservation service model. Diabetes Care. $2005 ; 28(2): 248-53$

31. Edmonds ME, Blundell MP, Morris ME, Thomas EM, Cotton LT, Watkins PJ. Improved survival of the diabetic foot: the role of a specialized foot clinic. Q J Med. 1986;60(232):763-71.

32. Holstein P, Ellitsgaard N, Olsen BB, Ellitsgaard V. Decreasing incidence of major amputations in people with diabetes. Diabetologia. 2000;43(7):844-7.

Endereço para correspondência:

Karla Freire-Rezende

Av. Beira Mar, 1800, apart. 701 - Edifício Pallais de Versailles

- Praia 13 de Julho

49025-040, Aracaju SE

E-mail: kfr@oi.com.br 